Journal of Agriculture and Environmental Sciences June 2018, Vol. 7, No. 1, pp. 116-126

ISSN: 2334-2404 (Print), 2334-2412 (Online)

Copyright (C) The Author(s). All Rights Reserved.

Published by American Research Institute for Policy Development

DOI: $10.15640 /$ jaes.v7n1a12

URL: https://doi.org/10.15640/jaes.v7n1a12

\title{
Cereal crops choices and economic resilience face to climate variability in southern Mali
}

\author{
Keba SISSOKO$^{1}$, Kimseyinga SAVADOGO ${ }^{2}$, Michel VAKSMANN ${ }^{3}$ \& Abel TIEMTORE $^{4}$
}

\begin{abstract}
:
This study uses the production function approach to assess and compare resistance of millet, Sorghum and Maize facing climate variability in Sikasso and Ségou regions. A robust estimation of the multiple regression models with Ordinary Least Squares (OLS) method was used. Overall, the results show that: one of the major causes of the fluctuations of the chosen cereals' production is climate variability; cereals have different resistance level facing climate variability across regions; finally cereals are more related to climate in Sikasso region than Ségou region. At the sight of these results, setting up following policies was recommended: promotion of production system based on new agricultural practices to increase productivities in different areas and policies which promote ideal cultures for each region's agricultural development (regional specialization).
\end{abstract}

Key words: production function, robust estimation, climate variability and regional specialization.

JEL classifications: C22, Q16, Q18, Q54

\section{Introduction}

Le changement climatique affecte le secteur agricole de tous les pays du monde mais particulièrement ceux de l'Afrique subsaharienne car on y pratique essentiellement une agriculture de subsistance. Dans cette zone en générale et particulièrement au Mali, l'agriculture est de type pluvial, très extensif et peu mécanisé. Les scénarios climatiques du Mali à l'horizon 2025 prévoient une diminution de la pluviométrie avec des taux de pertes par rapport à la normale de 2 à $6 \%$ et une augmentation des températures de $1{ }^{\circ} \mathrm{C}$ par rapport à la normale. La pollution dans le pays représente $0.06 \%$ de l'émission totale de gaz à effet de serre (GES) dans le monde (CCNUCC 5 , 2015). Au regard des statistiques, on constate une forte baisse de la pluviométrie sur les 50 dernières années. Entre 1951 et 1970, la pluviométrie annuelle atteignait $95 \mathrm{~mm}$ à Tessalit au Nord et $1360 \mathrm{~mm}$ à Sikasso au Sud. Tandis qu'entre 1971 et 2000, la pluviométrie est passée à $70 \mathrm{~mm}$ à Tessalit et $1181 \mathrm{~mm}$ à Sikasso soit une baisse de $24 \mathrm{~mm}$ au Nord et $179 \mathrm{~mm}$ au Sud en seulement un demi-siècle (source $\mathrm{DNM}^{6}$ ).

Au Mali, l'agriculture s'exerce dans des conditions climatiques aléatoires avec des risques importants de sécheresse. Elle subit par conséquent des fluctuations importantes liées à la mauvaise répartition des pluies dans le temps. C'est pourquoi au cours des 20 dernières années, sans sécheresse très prononcée, la production agricole a varié du simple au double entre la plus mauvaise campagne et la meilleure. Cette variabilité interannuelle et inter-saisonnière est l'un des principaux facteurs de vulnérabilité des producteurs (CILSS, 2002)

L économie du Mali est, de par ses caractéristiques actuelles, très exposée aux risques climatiques. En 2010, le secteur agricole occupait plus de $80 \%$ de la population active et représentait 38,5\% du produit intérieur brut (PIB), alors que le secteur industriel représentait 16,9\% du PIB et le secteur tertiaire (commerce, services) 37,6\% (CSCRP7, 2010).

\footnotetext{
${ }^{1}$ Assistant vacataire à l'Université des Sciences Sociales et de Gestion, Mali/sissokokeba11@yahoo.fr

2 Professeur, Université Ouaga 2, Ouagadougou, Burkina Faso/ksavadogo101@yahoo.com

${ }^{3}$ Chercheur, CIRAD, UMR AGAP, BP 1813, Bamako, Mali michel.vaksmann@cirad.fr

${ }^{4}$ Professeur, Université Ouaga 2, Ouagadougou, Burkina Faso/abel_tiemtore@yahoo.ca

${ }^{5}$ Convention Cadre des Nations Unies sur les Changements Climatiques

${ }^{6}$ Direction Nationale de la Météorologie

${ }^{7}$ Cadre Stratégique pour la Croissance et la Réduction de la Pauvreté
} 
La part du secteur agricole est en baisse car elle ne représentait que 30\% du PIB en 2012 (INSTAT ${ }^{8}$, 2013). Selon la Banque mondiale en 2014, plus de 55,6\% de la population vivait en deçà du seuil de pauvreté national et le taux d'insécurité alimentaire était de $25 \%$ en février 2015 selon l'ENSAN ${ }^{9}$. Cette situation est accompagnée par la série de crises que connait le pays à savoir : pastorale en 2010, sécheresse en 2011 et politico-sécuritaire à partir de 2012.

Les céréales les plus produites et consommées au Mali sont : le riz, le mil, le sorgho et le maïs. Les rendements respectifs de ces céréales sont en deçà de leurs rendements potentiels (CSA $\left.{ }^{10}, 2011\right)$. Sur la période 20012010, une augmentation considérable des superficies cultivées des principales céréales au Mali et une fluctuation du rendement de ces dernières a été constatée avec : un maximum de $1343 \mathrm{~kg} / \mathrm{ha}$ et un minimum de $737 \mathrm{~kg} / \mathrm{ha}$ pour le sorgho, un maximum de $939 \mathrm{~kg} / \mathrm{ha}$ et un minimum de $510 \mathrm{~kg} / \mathrm{ha}$ pour le mil et un maximum de $2682 \mathrm{~kg} / \mathrm{ha}$ et un minimum de $1148 \mathrm{~kg} / \mathrm{ha}$ pour le maïs (EAC/CPS/SDR, 2014/2015). Globalement, la superficie des terres cultivées en céréale est passée de 3295387 ha en 2009/10 à 4501380 ha en 2014/15, ce qui représente une augmentation de $37 \%$ en termes de défrichement (CPS11/SDR/EACI, 2014/2015). L'évolution du climat au Mali fera baisser la disponibilité de nourriture pour tous les producteurs (petits, moyens et grands), mais les grands producteurs resteront auto-suffisants. Les petits producteurs et les producteurs moyens perdront progressivement leurs autosuffisances (Traoré, 2014).

Face à tous ces problèmes, les autorités maliennes ont développé des plans en vue d'aider les producteurs à travers l'adoption de la Politique Nationale sur les Changements Climatiques (PNCC), qui a abouti à l'élaboration d'une Stratégie Nationale des changements climatiques (SNCC) en 2011 et la promotion de la recherche dans le domaine. Cela en plus de l'intervention de certaines ONG locales et internationales dans le secteur agricole à travers des appuis aux petits producteurs. Malgré ces efforts, le problème de fluctuation de la productivité persiste et devient de plus en plus inquiétant. Le producteur moyen est obligé d'adopter des comportements ex antes et ex post pour réduire les effets du changement climatique sur sa productivité. Cela conduit à rechercher la réponse à la question suivante : Dans un contexte de changement climatique, comment un choix optimal des cultures céréalières peut-il contribuer à l'augmentation de la résilience économique des producteurs au Mali ? L'objectif global de ce papier est de montrer et comparer la différence de résistance au changement climatique du maïs, du mil et du sorgho dans les régions de Sikasso et Ségou. Il s’agira plus spécifiquement de :

- Déterminer la différence de résistance qui existe entre les principales céréales face au changement climatique dans les différentes zones d'études.

- Apprécier l'efficacité d'un choix optimal des cultures céréalières en termes de résilience économique des producteurs face au changement climatique.

Les hypothèses suivantes soutiennent cette étude :

- $\mathrm{H}_{1}$ : Les variables climatiques ont des effets significatifs sur le niveau de production des céréales dans les différentes zones climatiques;

- $\mathrm{H}_{2}$ : Un choix optimal des cultures céréalières permet de stabiliser le niveau de production des céréales face à l'évolution du climat dans les différentes zones climatiques.

La seconde partie de ce document présente l'évolution du climat au Mali, les déterminants du choix des céréales et le cadre conceptuel; les matériels et la méthode sont exposés dans la troisième partie ; la quatrième partie est consacrée au modèle, résultats et discussions ; enfin la dernière partie présente les conclusions.

\section{Evolution du climat au Mali et déterminants du choix des céréales}

\subsection{Evolution du climat au Mali}

Des changements dans les fréquences des phénomènes météorologiques et climatiques extrêmes ont été observés depuis 1950. Ces phénomènes se manifestent par une forte variabilité de la pluviométrie, une augmentation des températures moyennes, une recrudescence des phénomènes climatiques extrêmes comme les inondations, sécheresses, etc. Dans les travaux du GIEC, le terme « changement climatique fait référence à tout changement dans

\footnotetext{
${ }^{8}$ Institut National de la Statistique

${ }^{9}$ Enquête Nationale sur la Sécurité Alimentaire et Nutritionnelle

${ }^{10}$ Commissariat à la Sécurité Alimentaire

11 Cellule de la Planification et de la Statistique
} 
le temps, qu'il soit dû à la variabilité naturelle ou aux activités humaines ». Par contre, dans la convention-cadre des Nations Unies sur les changements climatiques, le terme désigne uniquement les changements dus aux activités humaines. La convention cadre utilise le terme "variabilité climatique » pour désigner les changements climatiques d'origines naturelles (GIEC'12, 2014).

Au Mali, on rencontre quatre types de climat: le climat désertique au nord avec une pluviométrie annuelle inférieure à $200 \mathrm{~mm}$; le climat sahélien au centre avec une pluviométrie annuelle comprise entre 200 et $600 \mathrm{~mm}$; le climat soudanien plus au sud avec une pluviométrie annuelle comprise entre 600 et $1000 \mathrm{~mm}$ et le climat nord-guinéen observé à l'extrême sud du Mali avec une pluviométrie comprise entre 1000 et $1200 \mathrm{~mm}$. La mousson se compose des lignes de grain caractéristiques du Sahel orientée nord-sud et se déplaçant d'est en ouest s'accompagnant souvent de vents forts et de pluies abondantes parfois catastrophiques; une augmentation des températures du Sud-ouest vers le Nord-est avec des maximales relevées au cours de l'année pouvant atteindre ou dépasser les $40^{\circ} \mathrm{C}$ tandis que les minimales sont rarement en dessous de $18^{\circ} \mathrm{C}$; de fortes valeurs de l'évapotranspiration potentielle (ETP) en raison des températures élevées, des humidités relativement faibles et des vents forts

Une baisse de la pluviométrie a été constatée à partir des années 1970 accompagnée d'une grande variation spatiotemporelle de la pluviométrie ; entraînant des déficits pluviométriques assez importants et un déplacement des isohyètes vers le sud, ce qui fait que la migration est devenue de plus en plus une stratégie face à ces nouvelles conditions climatiques et environnementales précaires. On y note de nos jours en moyenne une baisse de $20 \%$ de la pluviométrie entre la période 1951-1970 (période humide) et la dernière période de référence 1971-2000 entraînant un déplacement des isohyètes de $200 \mathrm{~km}$ vers le Sud. L'isohyète $1200 \mathrm{~mm}$ n'existe plus sur la carte du Mali (PANA, 2007).

Les températures seraient en hausse sur l'ensemble du pays de plus de $2^{\circ} \mathrm{C}$ avec une perception de chaleur forte. On prévoit une diminution de la pluviométrie par rapport à la normale 1961-1990 ce qui entraînerait une situation de sécheresse au Mali comparable à celle des années 1970 (Projet Climat/CNRST, 2003). Cette situation serait constatée sur la première moitié de l'hivernage (mai, juin et juillet) à partir de l'horizon 2025 et elle pourrait s'installer dès l'horizon 2020 si la réaction du climat aux perturbations devenait plus rapide (Traoré et al., 2004).

La date de début des pluies est plus variable que la date de fin de l'hivernage au Mali. La durée suit un gradient régressif du sud vers le nord allant de 140 à 60 jours. Plus on se déplace vers le nord, plus la durée de la saison est courte (Soumaré et al, 2006). D’un autre côté on note que les sécheresses des années 1970 et 1980 n'ont pas entraîné de changements profonds de la longueur de la saison des pluies sur les 30 dernières années (Sissoko et al., 2008). La baisse de la pluviométrie n'est ni continue dans le temps ni homogène dans l'espace. Les épisodes secs et moins secs ne sont pas intervenus aux mêmes moments selon la latitude (Soumaré et al., 2008). L'analyse des effets liés à la variabilité climatique et au changement climatique sur la production de céréales à N’Tarla a permis de montrer que sur la période 1965-2005, les températures maximales sont restées relativement (statistiquement) constantes (Traoré et al., 2013).

\subsection{Les déterminants du choix des céréales}

Le choix des céréales au Mali dépend des systèmes de productions qui diffèrent selon les zones et les caractéristiques des céréales. Selon les résultats de l'enquête menée en 2007 dans le cadre du programme RuralStruc, dans la zone de Macina (dans l'Office du Niger), le produit brut agricole repose presque exclusivement sur le riz $(78 \%)$ et l'échalote $(18 \%)$. À Koutiala, en zone cotonnière, une part non négligeable du produit brut est constituée avec le maïs $(15 \%)$; le coton occupe une place encore importante $(27 \%)$ même si les superficies cultivées ont baissé par rapport aux années précédentes ; le reste est constitué par le mil (22\%) et le sorgho (24\%) (Traoré et al., 2011).

La sélection variétale du sorgho a abouti à des variétés productives, mais qui se révèlent peu adaptées aux systèmes de culture extensifs, d'où la faible adoption de ces variétés par les paysans du Mali. Les variétés locales de sorgho sont bien adaptées à l'extrême variabilité du climat par leur caractère de sensibilité à la photopériode. Ce caractère leur permet de moduler naturellement la durée de leur cycle en fonction de la durée probable de la saison des pluies. Lorsque le semis est réalisé tôt, la durée de la période végétative se rallonge de façon à toujours grouper la floraison dans les 20 jours qui précèdent la fin des pluies moyennes (Kouressy et al., 2008). Le photopériodisme confère aux variétés obtenues une grande plasticité phénologique, le matériel demeure performant quelque soit la date de semis. Toutefois, cette caractéristique a été éliminée par la plupart des programmes d'amélioration du sorgho malgré la grande souplesse d'adaptation qu'elle confère à la culture (Vaksmann et al., 1996).

${ }^{12}$ Groupe Intergouvernemental d'Experts sur l'Evolution du Climat 


\section{Cadre conceptuel}

Plusieurs méthodes sont utilisées dans la littérature pour mesurer l'effet de la variabilité climatique sur la production agricole (Bazzaz et Sombroek, 1997). Parmi ces approches, nous avons l'approche par la fonction de production et l'approche Ricardienne. En Tunisie, Jeder et al. (2013) ont procédé à l'évaluation de la vulnérabilité de l'agriculture dans la plaine de Jeffara (sud-est tunisien) au changement climatique en utilisant la méthode Ricardienne. Les résultats ont permis de montrer que le climat a un effet non linéaire et significatif sur le revenu agricole. Par contre le modèle utilisé a montré des limites pour exprimer les comportements d'adaptation des agriculteurs même si, il a pu estimer la contribution positive de certains facteurs d'adaptation à l'amélioration du revenu agricole comme la pratique d'élevage, l'irrigation et l'accès à la vulgarisation.

Une étude au Burkina a permis de quantifier l'impact du changement climatique sur les revenus agricoles. Elle a montré que le climat a un effet non linéaire sur le revenu agricole et que l'agriculture est plus sensible au changement des précipitations qu'à celui de la température. Les simulations de cette étude montrent que les Scenarios de diminution des précipitations et d'augmentation des températures seront très dommageables à l'agriculture car les conditions climatiques sont déjà difficiles (Ouédraogo et al., 2012).

Une étude récente menée en Tunisie par Chebil et al. (2011) a analysé l'impact du changement climatique sur la productivité des cultures céréalières dans la région de Béja, située au nord-ouest de la Tunisie en utilisant l'approche par la fonction de production. Les variables climatiques (pluviométrie, température) et le progrès technique ont été utilisés comme déterminants du rendement des différentes cultures. Les résultats ont montré que :le rendement des blés dépendait des variables climatiques et du progrès technique ; l'influence de la pluviométrie et de la température sera encore plus importante jusqu'à l'horizon 2030 ; le blé tendre subira une vulnérabilité plus forte que les autres cultures et la région pourra enregistrer en 2030 des pertes en rendement céréalier de l'ordre de 2,04\%, 9,62\% et 6,78\% respectivement pour le blé dur, le blé tendre et l'orge. Cette approche a aussi été utilisée par Reilly et al. (1994), Rosenzweig et Iglesias (1994), Rosenzweig et Parry (1994), pour mesurer l'impact de la variabilité climatique sur la production végétale.

L'approche par la fonction de production cadre avec nos objectifs car c'est une approche expérimentale qui permet de mesurer les effets directs des facteurs de production sur le niveau de production. Elle est fondée sur l'existence d'une fonction de production pour toute culture, qui relie la production (ou le rendement) de la culture à son environnement biophysique. Cette approche estime directement le changement du rendement à partir des modèles de réponse des cultures. Elle permet d'estimer l'impact des changements climatiques sur le rendement, en faisant varier les niveaux des stimuli climatiques. Ce concept est dérivé de la théorie microéconomique du producteur, qui stipule que le consommateur cherche toujours à maximiser sa production sous contrainte de la technologie et de la quantité de chaque facteur de production.

\section{Matériels et méthodes}

\subsection{Sources des données}

Les données agronomiques telles que la superficie, la production à l'hectare des différentes cultures proviennent de la CPS/SDR (Cellule de la Planification et de la Statistique du Secteur du Développement Rural) et celles sur les variables climatiques (précipitations, températures des mois couvrant la saison des pluies, dates de début et de fin des pluies) proviennent de la direction nationale de la météorologie (DNM). Il s'agit de séries qui portent sur au moins 30 ans dans deux régions du Mali. A Sikasso, les variables climatiques retenues sont les précipitations et températures des mois de Mai à Octobre tandis qu’à Ségou, les variables climatiques sont les précipitations et températures des mois de juin à octobre. Dans le cadre de cette étude, nous n'avons considéré que les températures minimales car les températures maximales sont restées relativement constantes sur la période d'étude.

\subsection{Traitement et méthode d'analyse des données}

Les séries de données brutes enregistrées ont été vérifiées pour voir la cohérence des données collectées. Les données incohérentes ou douteuses ont été corrigées ou remplacées par des données vraisemblables suivant les techniques d'usage de nettoyage des bases de données (technique de la moyenne ou la proportion des valeurs consécutives) pour éviter les données manquantes. Des analyses graphiques ont aussi été effectuées pour détecter les corrélations fallacieuses et repérer les données aberrantes.

\subsection{Couverture spatiale}

Le Mali est un pays vaste et comporte diverses zones climatiques. Les caractéristiques climatiques et 
pédologiques diffèrent d'une région à une autre sur les huit régions administratives. Les données couvrent une période de 31 ans (1980-2010) pour Sikasso et 30 ans pour Ségou. La période 1961-1990 est considérée par l'Organisation Mondiale de la Météorologie (OMM) comme la dernière normale climatique. Toute période située au-delà de cette période de référence correspond donc à une période de manifestation du changement ou de la variation climatique. Les périodes choisies dans le cadre de cette étude se situent donc entre les deux périodes de références climatiques.

\subsection{Procédures d'estimation du modèle}

Le test de rang de Szroeter (1978) a été effectué pour déterminer les variables responsables de l'hétéroscedasticité dans la base. Un autre problème de spécification du modèle a aussi été détecté grâce au test de Ramsey. Ce problème est dû aux objectifs de cette étude qui ne considère que les variables climatiques dans le modèle. Les modèles de régression multiples ont été estimés par la méthode des Moindres Carrés Ordinaires avec l'option robuste pour corriger le problème d'hétéroscedasticité. Il s'agit d'un modèle de régression multiple pour chaque céréale et dans chacune des deux zones. Les variables climatiques sont exogènes pour les trois céréales dans chaque zone d'étude afin de capter l'effet des variabilités climatiques sur la production des céréales.

\subsection{Les modèles}

La forme fonctionnelle non spécifiée des fonctions de production estimées dans le cadre de cette étude est linéaire. Il se présente comme suit :

$$
\ln \left(Y_{t}\right)=\beta_{0}+\sum_{t=1}^{n} \beta_{i t} \ln \left(X_{i t}\right)+\varepsilon_{t} \quad \mathrm{i}: 1 \ldots \ldots \mathrm{m}
$$

$\ln \left(Y_{t}\right)$ : Les variables endogènes en logarithme ;

$\beta_{0}:$ La constante ;

$\beta_{i}:$ Le vecteur des coefficients des variables exogènes;

$\ln \left(X_{i}\right)$ : Le vecteur des variables exogènes en logarithme;

$\varepsilon_{t}:$ Terme d'erreur, i: le nombre de variables exogènes et $t$ : les années.

$\beta_{i}=d \ln (Y) / d \ln \left(X_{i}\right)$ : L'élasticité de la variable endogène par rapport aux variables exogènes.

En prenant en compte les variables explicatives, on obtient la forme spécifiée suivante :

$\ln \left(Y_{t}\right)=f\left(\beta_{0}, \ln \left(\right.\right.$ plu $\left._{t}\right), \ln \left(\right.$ temps $\left._{t}\right), \ln \left(\right.$ debut $\left._{t}\right), \ln \left(\right.$ fin $\left.\left._{t}\right)\right)$

$\ln \left(Y_{t}\right):$ Logarithme de la production de céréales ;

$\beta_{0}:$ La constante;

$\ln \left(p l u_{t}\right):$ Logarithme des pluviométries par mois pendant l'hivernage;

$\ln \left(\right.$ temps $\left._{t}\right)$ : Logarithme des températures par mois pendant l'hivernage;

$\ln \left(\right.$ début $\left._{t}\right)$ : Logarithme des dates de début des pluies ;

$\ln \left(\right.$ fin $\left._{t}\right):$ Logarithme des dates de fin des pluies et $t$ : Années.

L'estimation est faite par céréales et par zone d'étude. Les deux modèles pour les trois céréales à Sikasso et Ségou sont respectivement : 


$$
\begin{aligned}
& \ln \left(Y_{t}\right)=\beta_{0}+\beta_{1} \ln \left(p l u_{m a t}\right)+\beta_{2} \ln \left(p l u_{j n t}\right)+\beta_{3} \ln \left(p l u_{j l t}\right)+\beta_{4} \ln \left(p l u_{a o t}\right)+\beta_{5} \ln \left(p l u_{s p t}\right) \\
& +\beta_{6} \ln \left(\text { plu }_{\text {oct }}\right)+\beta_{7} \ln \left(\text { temps }_{\text {mat }}\right)+\beta_{8} \ln \left(\text { temps } s_{\text {jnt }}\right)+\beta_{9} \ln \left(\text { temps } s_{j l t}\right)+\beta_{10} \ln \left(\text { temps } s_{\text {aot }}\right) \\
& +\beta_{11} \ln \left(\text { temps }_{\text {spt }}\right)+\beta_{12} \ln \left(\text { temps }_{\text {oct }}\right)+\beta_{13} \ln \left(\text { dédut }_{t}\right)+\beta_{14} \ln \left(\text { fin }_{t}\right)+\varepsilon_{t} \\
& \ln \left(Y_{t}\right)=\beta_{0}+\beta_{1} \ln \left(p l u_{j n t}\right)+\beta_{2} \ln \left(p l u_{j l t}\right)+\beta_{3} \ln \left(p l u_{\text {aot }}\right)+\beta_{4} \ln \left(p l u_{s p t}\right)+\beta_{5} \ln \left(p l u_{o c t}\right) \\
& +\beta_{6} \ln \left(\text { temps }_{j n t}\right)+\beta_{7} \ln \left(\text { temps }_{j l t}\right)+\beta_{8} \ln \left(\text { temps }_{\text {aot }}\right)+\beta_{9} \ln \left(\text { temps }_{\text {spt }}\right)+\beta_{10} \ln \left(\text { temps } s_{\text {oct }}\right) \\
& +\beta_{11} \ln \left(\text { dédut }_{t}\right)+\beta_{12} \ln \left(\text { fin }_{t}\right)+\varepsilon_{t}
\end{aligned}
$$

Avec :

$\ln \left(Y_{t}\right)$ : Logarithme des productions de maïs, sorgho et mil;

$\beta_{0}$ : Constantes ;

$\beta$ : Coefficients des variables climatiques;

$\ln \left(p l u_{\text {mat }}\right)$ : Logarithme de la pluviométrie du mois de mai ;

$\ln \left(p l u_{j n t}\right):$ Logarithme de la pluviométrie du mois de juin ;

$\ln \left(p l u_{j l t}\right)$ : Logarithme de la pluviométrie du mois de juillet ;

$\ln \left(p l u_{a o t}\right)$ : Logarithme de la pluviométrie du mois d'août ;

$\ln \left(p l u_{s p t}\right):$ Logarithme de la pluviométrie du mois de septembre ;

$\ln \left(p l u_{\text {oct }}\right)$ : Logarithme de la pluviométrie du mois d'octobre ;

$\ln \left(\right.$ temps $\left._{\text {mat }}\right)$ : Logarithme de la température du mois de mai ;

$\ln \left(\right.$ temps $\left._{\text {jnt }}\right):$ Logarithme de la température du mois de juin ;

$\ln \left(\right.$ temps $\left._{j l t}\right):$ Logarithme de la température du mois de juillet ;

$\ln \left(\right.$ temps $\left.s_{a o t}\right)$ : Logarithme de la température du mois d'août ;

$\ln \left(\right.$ temps $\left._{\text {spt }}\right)$ : Logarithme de la température du mois de septembre ;

$\ln \left(\right.$ temps $\left._{\text {oct }}\right)$ : Logarithme de la température du mois d'octobre ;

$\ln \left(\right.$ début $\left._{t}\right)$ : Logarithme des Dates de début des pluies ;

$\ln \left(\right.$ fin $\left._{t}\right)$ : Logarithme des Dates de fin des pluies;

$\varepsilon_{t}:$ Terme d'erreur et $t:$ Années.

\section{Résultats et discutions}

Les résultats de l'estimation pour les modèles de la région de Sikasso sont présentés dans le tableau n ${ }^{\circ}$. 
Tableau ${ }^{\circ} 1$ : Résultat de l'estimation pour Sikasso

\begin{tabular}{|c|c|c|c|}
\hline \multirow[b]{2}{*}{$\begin{array}{l}\text { Variables } \\
\text { exogènes }\end{array}$} & \multicolumn{3}{|c|}{ Variables endogènes } \\
\hline & $\begin{array}{l}\ln \text { (Production de } \\
\text { maïs) }\end{array}$ & $\begin{array}{l}\text { ln (Production de } \\
\text { sorgho) }\end{array}$ & $\begin{array}{l}\text { ln (Production } \\
\text { de mil) }\end{array}$ \\
\hline $\ln \left(\mathrm{Plu}_{\mathrm{mat}}\right)$ & $\begin{array}{l}-0,699 * * \\
(-2,31)\end{array}$ & $\begin{array}{l}-0,372^{* *} \\
(-2,18)\end{array}$ & $\begin{array}{l}-0,551^{* *} \\
(-2,23)\end{array}$ \\
\hline $\ln \left(\mathrm{Plu}_{j \mathrm{nt}}\right)$ & $\begin{array}{l}0,0238 \\
(-0,04)\end{array}$ & $\begin{array}{l}0,0394 \\
(+0,12)\end{array}$ & $\begin{array}{l}-0,305 \\
(-0,77)\end{array}$ \\
\hline $\ln \left(\mathrm{Plu}_{\mathrm{jlt}}\right)$ & $\begin{array}{l}-0,784 \\
(-1,04)\end{array}$ & $\begin{array}{l}-0,356 \\
(-0,92)\end{array}$ & $\begin{array}{l}-0,723 \\
(-1,31)\end{array}$ \\
\hline $\ln \left(\mathrm{Plu}_{\mathrm{aot}}\right)$ & $\begin{array}{l}0,363 \\
(+0,62)\end{array}$ & $\begin{array}{l}0,0125 \\
(+0,04)\end{array}$ & $\begin{array}{l}0,379 \\
(+0,81)\end{array}$ \\
\hline $\ln \left(\mathrm{Plu}_{\mathrm{spt}}\right)$ & $\begin{array}{l}0,596 \\
(+0,93)\end{array}$ & $\begin{array}{l}0,158 \\
(+0,61)\end{array}$ & $\begin{array}{l}0,182 \\
(+0,44)\end{array}$ \\
\hline $\ln \left(\mathrm{Plu}_{\mathrm{oct}}\right)$ & $\begin{array}{l}0,259 \\
(+1,25)\end{array}$ & $\begin{array}{l}0,224 * * \\
(+2,22)\end{array}$ & $\begin{array}{l}0,174 \\
(+1,31)\end{array}$ \\
\hline $\ln \left(\right.$ temps $\left.s_{\text {mat }}\right)$ & $\begin{array}{l}-2,45 \\
(-0,57)\end{array}$ & $\begin{array}{l}-1,72 \\
(-0,55)\end{array}$ & $\begin{array}{l}0,633 \\
(+0,13)\end{array}$ \\
\hline $\ln \left(\right.$ temps $\left.s_{\text {int }}\right)$ & $\begin{array}{l}21,11^{*} \\
(+2,03)\end{array}$ & $\begin{array}{l}8,586^{*} \\
(+1,76)\end{array}$ & $\begin{array}{l}5,055 \\
(+0,64)\end{array}$ \\
\hline $\ln \left(t_{e m p s} \mathrm{j}_{\mathrm{jt}}\right)$ & $\begin{array}{l}-0,947 \\
(-0,07)\end{array}$ & $\begin{array}{l}0,781 \\
(+0,11)\end{array}$ & $\begin{array}{l}-5,757 \\
(-0,49)\end{array}$ \\
\hline $\ln ($ tempsaot $)$ & $\begin{array}{l}-2,981 \\
(-0,21)\end{array}$ & $\begin{array}{l}-4,124 \\
(-0,57)\end{array}$ & $\begin{array}{l}-9,564 \\
(-1,20)\end{array}$ \\
\hline $\ln \left(\right.$ temps $\left.s_{\text {spt }}\right)$ & $\begin{array}{l}-5,812 \\
(-0,40)\end{array}$ & $\begin{array}{l}-2,345 \\
(-0,34)\end{array}$ & $\begin{array}{l}-1,223 \\
(-0,12)\end{array}$ \\
\hline $\ln ($ tempsoct $)$ & $\begin{array}{l}14,06^{* *} \\
(+2,16)\end{array}$ & $\begin{array}{l}3,603 \\
(+0,97)\end{array}$ & $\begin{array}{l}8,056 \\
(+1,57)\end{array}$ \\
\hline $\ln \left(\right.$ début $\left._{\mathrm{t}}\right)$ & $\begin{array}{l}-0,652 \\
(-0,40)\end{array}$ & $\begin{array}{l}-0,474 \\
(-0,65)\end{array}$ & $\begin{array}{l}-0,49 \\
(-0,51)\end{array}$ \\
\hline $\ln \left(\mathrm{fin}_{\mathrm{t}}\right)$ & $\begin{array}{l}-1,129 \\
(-0,26)\end{array}$ & $\begin{array}{l}0,994 \\
(+0,62)\end{array}$ & $\begin{array}{l}2,569 \\
(+1,03)\end{array}$ \\
\hline Constante & $\begin{array}{l}-49,01 \\
(-1,13) \\
\end{array}$ & $\begin{array}{l}-4,562 \\
(-0,19)\end{array}$ & $\begin{array}{l}11,91 \\
(+0,33)\end{array}$ \\
\hline Observations & 31 & 31 & 31 \\
\hline R-carré & $0,50 * * *$ & $0,60 * * *$ & $0,49 * * *$ \\
\hline F-stat & 3,93 & 3,32 & 5,44 \\
\hline
\end{tabular}

Le modèle explique à hauteur de 50\% les variations de la production de maïs à Sikasso sur la période 19802010. On note spécifiquement qu'une augmentation d'un pourcent de la pluviométrie de mai entraine une diminution de la production de maïs de $0,69 \%$, qu'une augmentation d'un pourcent de la température de juin entraine une augmentation de la production de $21,11 \%$ et qu'une augmentation d'un pourcent de la température d'octobre entraine une augmentation de la production de $14,06 \%$. Le modèle explique à hauteur de $60 \%$ les variations de la production de sorgho à Sikasso sur la période 1980-2010. On note spécifiquement qu'une augmentation d'un pourcent de la pluviométrie de mai entraine une diminution de la production de maïs de $0,372 \%$, qu'une augmentation d'un pourcent de la pluviométrie d'octobre entraine une augmentation de la production de maïs de $0,22 \%$ et qu'une augmentation d'un pourcent de la température de juin entraine une augmentation de la production de maïs de 8,58\%. Le modèle explique à hauteur de 49\% les variations de la production de mil à Sikasso sur la période 1980-2010. On note 
spécifiquement qu'une augmentation d'un pourcent de la pluviométrie de mai entraine une diminution de la production de mil de $0,55 \%$.

Les résultats de l'estimation pour les modèles de la région de Ségou sont présentés dans le tableau nº 2 .

Tableau n² : Résultat de l'estimation pour Ségou

\begin{tabular}{|c|c|c|c|}
\hline \multirow[b]{2}{*}{$\begin{array}{l}\text { Variables } \\
\text { exogènes }\end{array}$} & \multicolumn{3}{|l|}{ Variables endogènes } \\
\hline & $\begin{array}{l}\ln \text { (Production de } \\
\text { maiis) }\end{array}$ & $\begin{array}{l}\text { ln (Production de } \\
\text { sorgho) }\end{array}$ & $\begin{array}{l}\ln \text { (Production de } \\
\text { mil) }\end{array}$ \\
\hline \multirow[t]{2}{*}{$\ln \left(\operatorname{Plu}_{\text {jnt }}\right)$} & $-0,0445$ & 0,133 & $-0,344 * *$ \\
\hline & $(-0,26)$ & $(+0,76)$ & $(-2,83)$ \\
\hline \multirow[t]{2}{*}{$\ln \left(\mathrm{Plu}_{\mathrm{jlt}}\right)$} & 0,046 & 0,0354 & $-0,0571$ \\
\hline & $(+1,18)$ & $(+0,13)$ & $(-0,36)$ \\
\hline \multirow[t]{2}{*}{$\ln \left(\mathrm{Plu}_{\mathrm{aot}}\right)$} & $1,149 * * *$ & $0,625^{* *}$ & $0,339 *$ \\
\hline & $(+4,9)$ & $(+2,56)$ & $(+2)$ \\
\hline \multirow[t]{2}{*}{$\ln \left(\mathrm{Plu}_{\mathrm{spt}}\right)$} & 0,455 & $-0,159$ & $0,394 * *$ \\
\hline & $(+1,61)$ & $(-0,70)$ & $(+2,48)$ \\
\hline \multirow[t]{2}{*}{$\ln \left(\mathrm{Plu}_{\mathrm{oct}}\right)$} & $-0,0539$ & $-0,0208$ & 0,0274 \\
\hline & $(-1,25)$ & $(-0,59)$ & $(+1,16)$ \\
\hline \multirow[t]{2}{*}{$\ln \left(\right.$ temps $\left.s_{\text {jnt }}\right)$} & $11,40^{* *}$ & $-0,044$ & $-2,19$ \\
\hline & $(+2,75)$ & $(-0,01)$ & $(-0,60)$ \\
\hline \multirow[t]{2}{*}{$\ln \left(\right.$ temps $\left._{j \mathrm{jt}}\right)$} & 8,941 & $-1,905$ & $-9,979 * *$ \\
\hline & $(+1,09)$ & $(-0,33)$ & $(-2,40)$ \\
\hline \multirow[t]{2}{*}{$\ln ($ tempsaot $)$} & $-11,86$ & $-7,31$ & $-8,146$ \\
\hline & $(-1,13)$ & $(-0,83)$ & $(-1,24)$ \\
\hline \multirow[t]{2}{*}{$\ln \left(\mathrm{temps}_{\mathrm{spt}}\right)$} & 6,689 & $-1,785$ & $16,80^{* *}$ \\
\hline & $(+0,6)$ & $(-0,17)$ & $(+2,38)$ \\
\hline \multirow[t]{2}{*}{$\ln ($ tempsoct $)$} & $9,847^{* *}$ & 3,547 & 2,258 \\
\hline & $(+2,18)$ & $(+0,86)$ & $(+0,87)$ \\
\hline \multirow[t]{2}{*}{$\ln \left(\right.$ début $_{\mathrm{t}}$ ) } & $-4,421 * * *$ & 0,395 & $-0,385$ \\
\hline & $(-3,86)$ & $(+0,33)$ & $(-0,55)$ \\
\hline \multirow[t]{2}{*}{$\ln \left(\mathrm{fin}_{\mathrm{t}}\right)$} & 2,613 & 3,459 & $-1,502$ \\
\hline & $(+0,67)$ & $(+0,98)$ & $(-0,63)$ \\
\hline \multirow[t]{2}{*}{ Constant } & $-70,12 * *$ & 10,46 & 25,44 \\
\hline & $(-2,40)$ & $(+0,45)$ & $(+1,39)$ \\
\hline Observations & 30 & 30 & 30 \\
\hline R-carré & $0,77 * * *$ & $0,43^{* *}$ & $0,50^{* * *}$ \\
\hline F-stat & 11,36 & 2,87 & 4,88 \\
\hline
\end{tabular}

Source : Auteur, sur estimation des données de la CPS/SDR et de la DNM (1980-2009)

*, ** et *** signifient respectivement les seuils de significativité à $10 \%$, 5\% et $1 \%$.

(.) : t-Student

Le modèle explique à hauteur de 77\% les variations de la production de maïs à Ségou sur la période 19802009. On note spécifiquement qu'une augmentation d'un pourcent de la pluviométrie d'août entraine une augmentation de la production de maïs de $1,15 \%$, qu'une augmentation d'un pourcent de la température de juin entraine une augmentation de la production de maïs de $11,40 \%$, qu'une augmentation d'un pourcent de la température d'octobre entraine une augmentation de la production de maïs de 9,847\% et qu'une augmentation (retard) d'un pourcent des dates de début entraine une diminution de la production de maïs de 4,421\%. Le modèle explique à hauteur de 43\% les variations de la production du mil à Ségou sur la période 1980-2009. On note spécifiquement qu'une augmentation d'un pourcent de la pluviométrie d'août entraine une augmentation de la production sorgho de 0,625\%. Le modèle explique à hauteur de 50\% les variations de la production de sorgho à Ségou sur la période 19802009. On note spécifiquement qu'une augmentation d'un pourcent de la pluviométrie de mai entraine une diminution de la production de mil de 0,344\%, qu'une augmentation d'un pourcent de la pluviométrie d'août entraine une augmentation de la production de mil de $0,339 \%$, qu'une augmentation d'un pourcent de la pluviométrie de septembre entraine une augmentation de la production de mil de 0,394\%, qu'une augmentation d'un pourcent de la température 
de juillet entraine une diminution de la production de mil de 9,979\% et qu'une augmentation d'un pourcent de la température de septembre entraine une augmentation de la production de mil de $16,80 \%$.

Une analyse des résultats de cette étude nous montre que : le mil est plus stable face à la variabilité climatique dans la région de Sikasso avec un taux de dépendance de 49\% que celle de Ségou, il est suivi par le maïs et le sorgho qui sont plus sensibles à la variabilité climatique que la première spéculation sur la période d'étude avec des taux de dépendance respectives de 50\% et 60\% ; le sorgho a plus résisté face à la variabilité climatique dans la région de Ségou avec un taux de dépendance de $43 \%$ que celle de Sikasso, il est suivi par le mil et le maïs qui sont plus sensibles à la variabilité climatique que la première spéculation avec des taux de dépendances respectives de 50\% et 77\% ; le maïs a plus résisté à la variabilité climatique dans la région de Sikasso que celle de Ségou ; le sorgho a plus résisté à la variabilité climatique dans la région de Ségou que celle de Sikasso ; le mil a plus résisté à la variabilité climatique dans la région de Sikasso que celle de Mopti; dans la zone soudano-guinéenne, la date de début et la date de fin des pluies n'ont pas influencé significativement la production des céréales sur la période d'étude, enfin dans la zone soudanienne, les dates de début des pluies ont eu une influence significative sur la production avec une élasticité de -4,42, alors que celles des fins des pluies n'ont pas eu d'influence significative. La baisse constante de la production de maïs à Ségou peut s'expliquer par l'abandon de cette céréale par les producteurs de la zone qui ont peurs des risques liés à cette culture.

Les céréales sont globalement plus liées à la variabilité climatique dans la région de Ségou que celle de Sikasso avec des taux de dépendances moyens respectifs de $60 \%$ et $53 \%$. Cela est dû au fait que le niveau annuel de la pluviométrie dans la région de Ségou est inférieur à celui de Sikasso.

La pluviométrie de mai, les températures de septembre et d'octobre sont déterminantes pour le maïs ; les pluviométries de mai et d'octobre sont déterminantes pour le sorgho et la pluviométrie de mai est déterminante pour le mil dans la région de Sikasso. La pluviométrie d'août, la température de juin, la température d'Octobre et les dates de début des pluies sont déterminantes pour le maïs ; la pluviométrie d'août est déterminante pour le sorgho et la pluviométrie de juin, la pluviométrie d'août, la pluviométrie de septembre, la température de juillet et la température de septembre sont déterminantes pour le mil dans la région de Ségou. La pluviométrie de mai impacte négativement la production de toutes les spéculations de cette étude dans la région de Sikasso (zone soudano-guinéenne). Celle d'Août impact négativement la production de toutes les spéculations de cette étude dans la région de Ségou (zone soudanienne). Cela peut s'expliquer par le niveau de la pluviométrie de ces différents mois dans les différentes zones d'études.

En somme, on remarque que les variables climatiques qui expliquent statistiquement la production varient selon les types de céréales et la région. On note qu'à Ségou, les producteurs craignant les risques liés à la culture du maïs consacrent $52 \%$ des superficies totales de céréale à la culture du mil alors que c'est la culture du sorgho qui s'adapte le mieux à l'évolution du climat de la zone. Tandis qu'a Sikasso, les producteurs consacrent $46 \%$ des superficies totales de céréale à la culture du maïs alors que le mil s'adapte mieux à l'évolution du climat de la zone. La variabilité de la date de début des pluies expliquent son impact sur la production de maïs dans la région de Ségou. Cela peut donc expliquer la forte dépendance de la production des céréales à l'évolution du climat dans les différentes zones et la faible adaptation des variétés améliorées. Le faible taux d'adoption des technologies «moderne» par les petits exploitants agricoles peut aussi expliquer cette forte dépendance à la variabilité climatique. La spécialisation régionale peut donc aider les producteurs des différentes régions à réduire l'impact de cette variabilité climatique sur leurs niveaux de production cela doit être suivi par l'adoption de variétés à cycle court dans la région de Ségou car la saison de pluie est plus courte dans cette zone. Un choix optimal des cultures céréalières permettrait de stabiliser la production de céréale dans les différentes zones. Ce choix doit être la prémisse à un choix variétal pour une stabilisation ou une augmentation de la production des céréales dans les deux zones d'étude.

On peut donc déduire que les résultats confirment les deux hypothèses émises au départ qui soutiennent cette étude à savoir : les variables climatiques expliquent le niveau de production du maïs, du mil et du sorgho dans les différentes régions et un choix optimal des cultures céréalières permet de stabiliser le niveau de production de ces céréales face à la variabilité climatique dans les différentes régions.

Ces résultats sont aussi conformes aux résultats empiriques de Chebil et al. 2011) et Sissoko (2008) qui ont montré une forte corrélation entre le climat et la production. Par contre d'autres résultats contraires demeurent comme ceux de Soumaré et al. (2008) ; Ouédrago et al. (2012), qui ont affirmé qu'il n’y a pas de corrélation significative entre le climat et le rendement des céréales et que la faible adoption des variétés modernes résulterait du risque plus grand que celles-ci ferait courir aux producteurs. 


\section{Conclusions}

L'objectif principal de cette recherche était de montrer et comparer la différence de résistance qui existe entre le maïs, le mil et le sorgho face à la variabilité climatique dans les régions de Sikasso et Ségou. L'approche par la fonction de production a été utilisée dans le cadre de cette étude. Les fonctions de production ont été estimées par la méthode des Moindres Carrés Ordinaires avec option robuste pour corriger le problème d'hétéroscédasticité dans la base.

Les résultats montrent que : le mil a plus résisté face à la variabilité climatique que le sorgho et le maïs dans la région de Sikasso sur la période d'étude ; le sorgho a plus résisté face à la variabilité climatique que le mil et le maïs dans la région de Ségou ; le maïs a plus résisté à la variabilité climatique dans la région de Sikasso que celle de Ségou ; le sorgho a plus résisté à la variabilité climatique dans la région de Ségou que celle de Sikasso ; le mil a plus résisté à la variabilité climatique dans la région de Sikasso que celle de Ségou ; dans la région de Sikasso, la date de début et la date de fin des pluies n'ont pas influencé significativement la production des céréales, enfin dans la zone soudanienne, les dates de début des pluies ont eu une influence significative sur la production, alors que celles des fins des pluies n'ont pas eu d'influence significative sur la production des céréales. Le niveau de résistance des céréales varie selon les zones climatiques et les types de céréales. Cela montre que les céréales n’ont pas les mêmes caractéristiques et que les conditions pédologiques diffèrent selon les zones.

Face à ces résultats, on peut donc affirmer que l'une des causes majeures des fluctuations des productions du maïs, du mil et du sorgho est la variabilité climatique. Ainsi, l'idée d'une spécialisation régionale peut aider les producteurs à réduire l'impact de la variabilité climatique sur les productions céréalières dans les zones d'études et cette spécialisation doit être suivie par des choix variétaux optimaux. Ils sont une confirmation scientifique du choix des petits producteurs au Mali qui en général ont peur des incertitudes en matière de productivité (ils risquent leur vie) et préfèrent stabiliser leurs productions en choisissant les cultures traditionnelles moins productives mais mieux adaptées à leurs conditions climatiques et l'évolution de leurs climats.

Pour réduire la forte dépendance au climat des cultures céréalières dans les différentes régions, les politiques suivantes pourraient être mises en place par les autorités :

- des politiques de promotion d'un système de culture qui repose sur de nouvelles techniques culturales en vue d'augmenter la productivité ;

- des politiques d'appuis aux centres de recherches pour le développement de procédés de transformation modernes en vue de valoriser les cultures idéales pour chaque zone d'étude (spécialisation régionale) suivies par la création de conditions qui facilitent l'accès au marché ;

- Et, des politiques de vulgarisation des variétés précoces dans la région de Ségou pour que les paysans puissent mieux s'adapter à la courte saison des pluies dans cette région.

Enfin, ce travail ne prend pas en compte certaines réalités et phénomènes qui peuvent avoir des effets directs sur la production de céréale à savoir : la fertilité des sols, l'évolution génétique des céréales, l'évolution des pratiques culturales (notamment l'intensification agricole), la durée de l'ensoleillement et vitesse du vent. Une extension de cette étude à d'autres régions et d'autres types de cultures peut également aider à faire des déductions plus intéressantes.

\section{Références}

\section{Articles}

Bazzaz F. et Sombroek W. 1997. Changements du climat et production agricole : Effets directs et indirects du changement des processus hydrologiques, pédologiques et physiologiques des végétaux, Polytechnica, FAO, Rome, 406p

Chebil, A., Mtimet, N., et Tizaoui, H. (2011) "Impact du changement climatique sur la productivité des cultures céréalières dans la région de Béja (Tunisie)". African Journal of Agricultural and Resource Economics, vol.6, $\mathrm{n}^{\circ} 2$, pp.144-54.

Jeder, H., Khalifa, A. B., et Sghaier, M. (2013) "Impact des changements climatiques sur l'agriculture dans la plaine de Jeffara sud-est tunisien". Journal of Agriculture and Environment for International Development (JAEID), vol.107, n², pp.229-242.

Kouressy, M., Traoré, S.B., Vaksmann, M., Grum, M., Maikano, I., Soumaré, M., Traoré, P.S., Bazile, D., Dingkuhn, M., Sidibé, A., (2008) “Adaptation des sorghos du Mali à la variabilité climatique”. Cahiers Agricultures 17, 95-100. 
Ouedraogo, M. (2012) “Impact des changements climatiques sur les revenus agricoles au Burkina Faso". Journal of Agriculture and Environment for International Development (JAEID), vol.106, nº1, pp.3-21.

Reilly J., Hohmann N. and Kane S. 1994. Climate change and agricultural trade: who benefits, who loses? Global Envir. Change 4: 24-36.

Rosenzweig C. and Iglesias A. 1994. Implications of Climate Change for International Agriculture: Crop Modeling Study. US Environmental Protection Agency. EPA 230-

Rosenzweig C. and Parry M.L. 1994. Potential impacts of climate change on world food supply. Nature 367: 133-138.

Soumaré, M., Vaksmann, M., Bazile, D., Kouressy, M., \& Diakité, C. H. (2006) "Adaptation des systèmes de production soudano sahéliens à la sécheresse et conséquence pour la sélection variétale: le cas des sorghos du Mali". Gestion Concertée des Ressources et de l'Environnement Geocorev.

Traoré, A., Soumaré, M., Bélières, J. F., et Hilhorst, T. (2011) "Quelles évolutions des systèmes de production céréaliers au Mali?”. Grain de sel.

Traore, B. (2014) "Climate change, climate variability and adaptation options in smallholder cropping systems of the Sudano-Sahel region in West Africa (Wageningen University)".

Traore, B., Corbeels, M., van Wijk, M. T., Rufino, M. C., \& Giller, K. E. (2013). "Effects of climate variability and climate change on crop production in southern Mali". European Journal of Agronomy, vol.49, pp.115-125.

Traoré, F., Bayoko, A., Konaté, S., Coulibaly, A., et Diarra, B. (2004) "Etude des perspectives de changement climatique au Mali”. In Mali Symp. Appl. Sci. (MSAS), Bamako, Mali, pp.196-205.

Vaksmann, M., Kouressy, M., Chantereau, J., Bazile, D., Sagnard, F., Touré, A., et Danté, A. (2008) "Utilisation de la diversité génétique des sorghos locaux du Mali". Cahiers Agricultures, vol.17 n², pp.140-145.

Vaksmann, M., Traoré, S. B., et Niangado, O. (1996) "Le photopériodisme des sorghos africains". Agriculture et développement, vol. 9, pp.13-18.

\section{Rapports}

Couveinhes, P. (2015, November). “Changer avec le climat!". Annales des Mines-Responsabilité et environnement (No. 4, pp. 3-4). FFE.

DE VIE, E. D. C. (2007) "Programme d'action national d'adaptation à la variabilité et au changement climatique (PANA DE LA REPUBLIQUE DU MALI)".

MEF, Cellule Technique CSLP : Rapport Synthèse de Revue du CSCRP 2007-2011 portant sur 2010.

Ministère de l'agriculture. (2013) "Recueil des statistiques du secteur du développement rural". pp. 27 et 28.

Ministère du développement rural. (2015) “Annuaire statistique 2014 du développement rural”. Pages 14 et 24.

Rapport de synthèse - MALI "Enquête Nationale sur la Sécurité Alimentaire et Nutritionnelle (ENSAN MALI Septembre 2015)"

Simonet, G. (2015) "Une brève histoire de l'adaptation: l'évolution conceptuelle au fil des rapports du GIEC (19902014)”. Natures Sciences Sociétés, (Supp. 3), pp. 52-64.

Stratégie Nationale Changement climatique (SNCC) - rapport Mali (2011), Consulting Services, EcoSecurities.

Toe, A. M. (2002) "Étude pilote sur les limites maximales de résidus de pesticides admissibles dans les produits agricoles dans trois pays sélectionnés du CILSS (Burkina Faso, Mali, Sénégal). Consultation nationale pour le Burkina Faso. Rapport technique: mission de consultation". Projet GCP/RAF/335/NET/FAO/CILSS Gestion des pesticides au Sahel. 